\title{
Physiotherapy provides significant benefits for patients with chronic urologic pain
}

\author{
Nelly Faghani \\ Co-founder, Pelvic Health Solutions
}

Cite as: Can Urol Assoc J 2018;12(6Suppl3):S171-4. http://dx.doi.org/10.5489/cuai.5328

\section{Introduction}

Symptoms of urinary urgency, frequency, and bladder/pelvic pain debilitate a staggering number of people around the world. Many are left frustrated with symptoms of unknown cause and go through years of ineffective treatments, leaving them scared and without hope. The average patient has been seeking treatment for at least 3-5 years and has seen about 5-7 specialists during this time. Chronic urologic pain is much more prevalent than previously thought and patients present to us with multiple debilitating comorbidities that significantly impact their quality of life. A physiotherapist with the appropriate training to treat pelvic dysfunction can help many of your patients. The following are lessons that I have learned over the years that will help urologists understand the role of physiotherapy as part of the multimodal approach to the management of chronic urologic pain.

\section{Practice tips}

\section{Tip 1: Consider pelvic floor dysfunction}

A tip to my urology colleagues would be to consider the pelvic floor muscles as a contributor to our patient's symptoms. Prior to examining the bladder (anterior vaginal wall or bimanual) or prostate, I would recommend assessment of the pelvic floor muscles for tone (resistance to passive stretching), tenderness, and pain. Are symptoms reproduced with your palpation? Understanding the female and male pelvic floor anatomy, and various layers (Figs. 1-4), can help identify pelvic floor dysfunction and recognize patients who would benefit from a referral to a pelvic floor physiotherapist.

\section{Tip 2: Take a biopsychosocial approach}

Physiotherapy is an integral part of the interdisciplinary team that must take a comprehensive framework within a biopsychosocial approach when treating chronic urologic

pain. The biopsychosocial approach systematically considers psychological and social factors in addition to the biological factors, taking into account their complex interactions in understanding our patients' health.

\section{Tip 3: Listen to their story}

The subjective assessment provides an opportunity to establish a positive therapeutic connection with our patients. This is a fundamental first step in therapeutic neuroscience education for patients who are experiencing pain. The use of motivational interviewing, active and reflective listening, enables our patients to know that we have understood their story. We need to take thorough past medical histories and ask appropriate questions pertaining not only to their pain, but also to their bladder, bowel, and sexual function. This will help us set suitable patient-centred goals. Gaining our patients' trust, maintaining their dignity, and showing them compassion and empathy are key factors in determining rehabilitation outcomes. Every patient is unique and his or her pain is complex; the mind-body connection plays a significant role.

\section{Tip 4: Use appropriate outcome measures}

It is important that physiotherapists use validated and reliable outcome measures as part of our assessment tools. These help to assess our patient's behaviours, attitudes, and beliefs, and will guide us in our treatment planning and evaluate if our approach is effective. There are many outcome measures available; however, I will be highlighting three.

First a bladder and bowel diary will evaluate a baseline of our patient's micturition and bowel habits and fluid intake/type. Typically a three-day diary is sufficient to get a good picture of their behaviours/habits during various times of the week. Once evaluated, fluid modification, bladder retraining, and urge inhibition techniques can help control and reduce urgency, frequency, and incomplete emptying. Constipation is a top priority to address because it can impact bladder functions as well as pelvic pain.

The Depression, Anxiety, Stress Scale (DASS) is a selfreport questionnaire that is not to be used alone as a diagnostic tool, but it helps inform treatment and to make appro- 


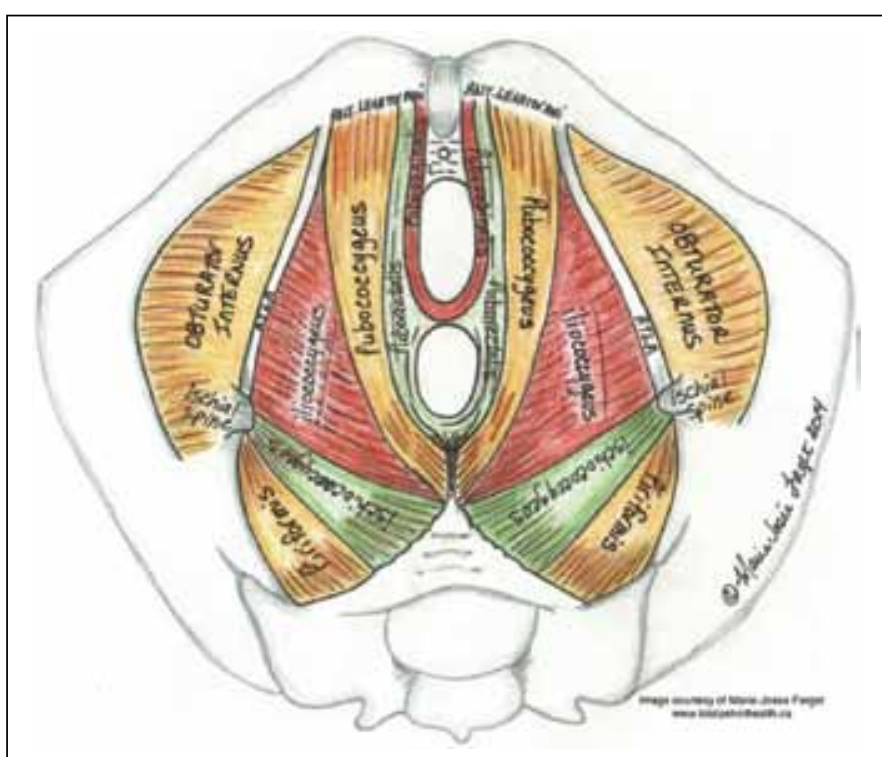

Fig. 1. Pelvic floor anatomy: Female layer 1.

priate outside referrals. The essential function of the DASS is to assess the severity of the core symptoms of depression, anxiety, and stress, which are highly correlated with pain.

The Pain Catastrophizing Scale (PCS) will help identify a psychosocial construct that is highly correlated with chronicity. There is Level 1 evidence that demonstrates that getting stuck in catastrophization is one of the strongest psychosocial predictors of chronicity, increased medication use, increased pain, and increased disability.

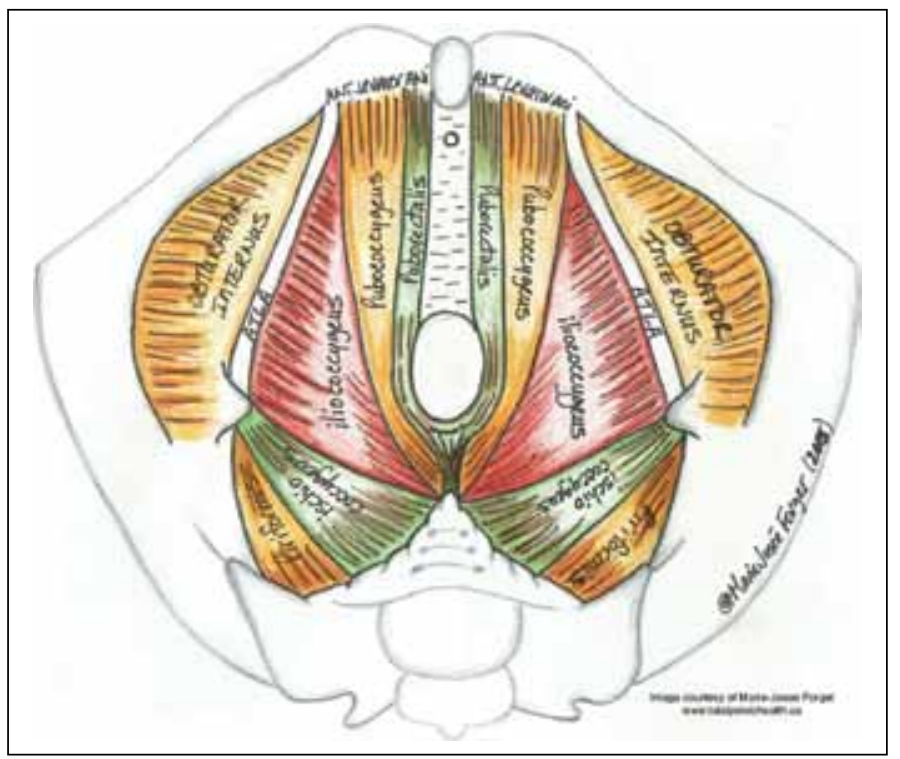

Fig. 3. Pelvic floor anatomy: Male layer 1.

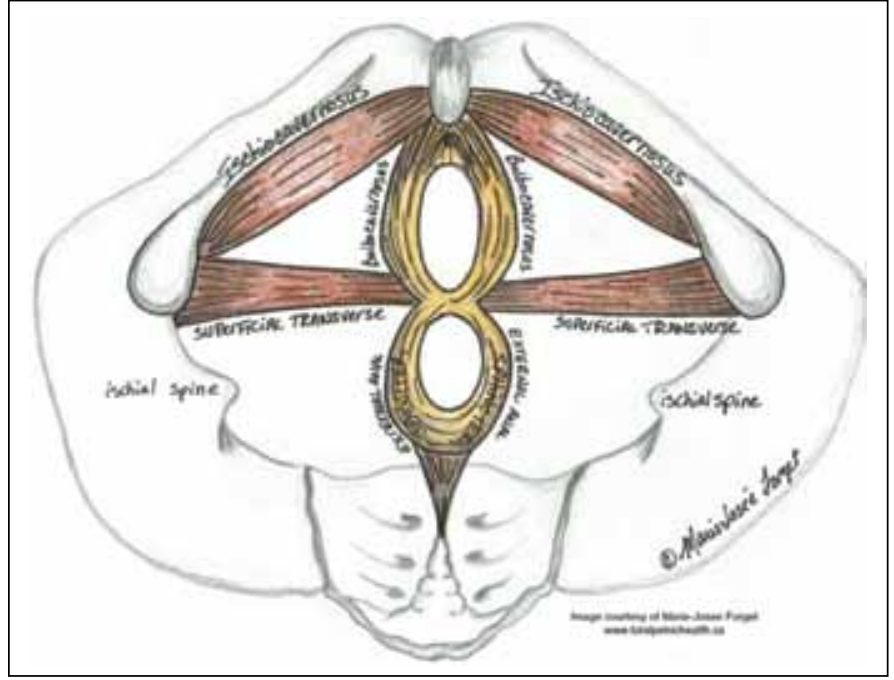

Fig. 2. Pelvic floor anatomy: Female layer 3.

Tip 5: Do a thorough objective evaluation

The objective physiotherapy assessment includes global assessment of respiratory function, posture and alignment, movement patterns, the lumbar and thoracic spine, connective tissue, neural involvement, and overall strength and conditioning. Pelvic floor physiotherapists have advanced training to assess the "state" of all three layers of the pelvic floor muscles (PFM) through external and internal vaginal and/or rectal palpation. The PFM's must be able to not only contract, but also relax for micturition, defecation, and even accommodation during sexual activity. Overactive pelvic floor muscles (OAPF) can be a significant contributor to our patients' pelvic pain. Overactivity of the PF is not simply an isolated local factor, but can be a physical manifestation

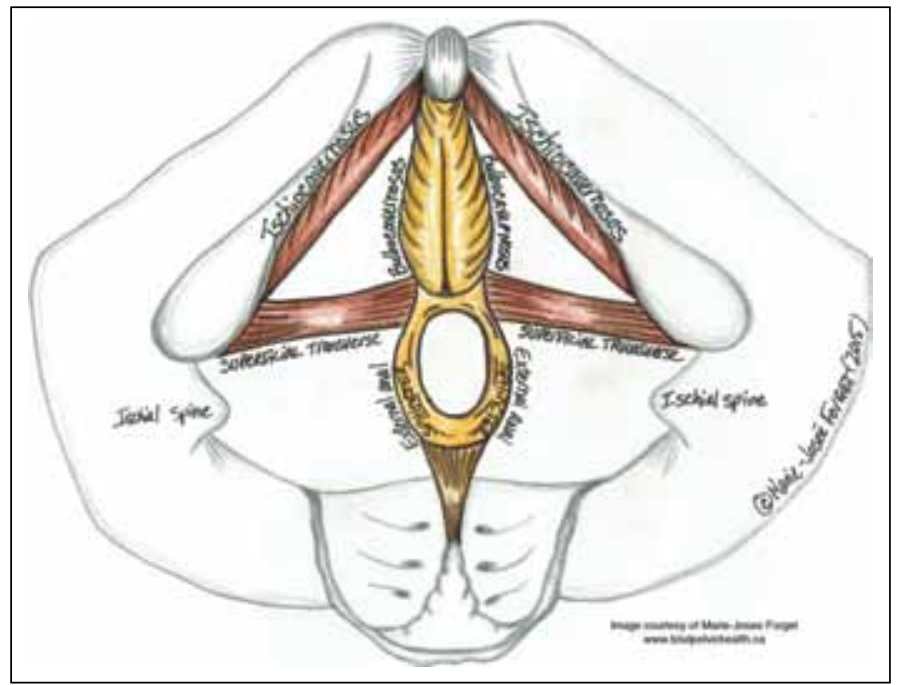

Fig. 4. Pelvic floor anatomy: Male layer 3. 
of our patients' emotional state. There are a variety of possible etiologies for the onset or existence of an OAPF, and it is important to identify what the predominant "drivers" or "threats" are in each person, and then develop an effective strategy to address these. Some of these etiologies include (but are not limited to) direct trauma (previous infection, childbirth or surgeries), psychological distress (anxiety, fear, or pain), psychosocial/psychosexual disturbances (trauma, abuse, poor relationships), postural patterns, abnormal behaviours, and/or patterns of pelvic floor use (constipation, frequent/delayed voiding).

\section{Tip 6: Therapeutic neuroscience education (TNE)}

Pain science research has evolved globally and now provides us credible evidence that we can treat and change the pain system through TNE. TNE reduces pain, disability, and catastrophization, and improves physical performance. This should be a first-line treatment.

By definition, pain is an unpleasant sensory and emotional experience associated with actual and potential tissue damage, or described in terms of such damage. It is always sensory and emotional. Pain is an output of the brain 100\% of the time. We need to validate our patients' pain experiences and readily reassure them that we know their pain is real. We also must explain to them that memories, emotions, thoughts, beliefs, attitudes, and especially expectations have an enormous influence on how pain is produced and how they perceive pain. Assessing and treating the emotional component needs to be considered in every patient. Thoughts are nerve impulses, and negative thinking alone can drive the pain.

We need to take the complex and make it simple through stories that help patients understand what is contributing to their symptoms.

\section{Tip 7: Diet modification}

We should recommend overall good nutrition, but one of the most common triggers for patients with chronic urologic pain is diet. Foods and beverages, such as caffeine, alcohol, carbonated beverages, tomato products, citrus fruits and juices, and cranberry juice are amongst the irritants that contribute to the intense discomfort and/or pain that our patient's experience. An elimination diet is the most effective way to help determine what foods need to be avoided.

\section{Tip 8: Breathing}

Breathing techniques are one of the first treatments we start because of the diaphragm's direct relationship with the pelvic floor. Shallow breathing does not allow for good diaphragmatic excursion, hence, contributing to the pelvic floor becoming rigid and overactive. Breathing is the only autonomic function of which we have voluntary control, and it is a fantastic and simple gateway into the autonomic system. This allows us to activate the parasympathetic system and calm down the powerful sympathetic system. Breathing is the most intuitive and inexpensive modality that can readily be taught to every patient by every member of the healthcare team. Diaphragmatic breathing with an extended exhale is one of the easiest interventions for our patients to learn, and can be prescribed for five minutes at a time, 5-6 times per day.

\section{Tip 9: Help improve their sleep}

More than $75 \%$ of people with persistent pain have disrupted sleep and $50-88 \%$ of people with pain have chronic insomnia, which would be compounded further with nocturia. There is a high correlation between disturbed sleep, depression, anxiety, catastrophization, fear avoidance behaviours, and general wellbeing. We must educate our patients on how poor sleep can impact their symptoms and how they can improve their sleep hygiene. Some important suggestions may include: sleeping at the same time every night, avoiding naps, staying away from lights (television and or handheld devices) at least one hour prior to bedtime, keeping the bedroom cool, and avoiding fluids in the evening.

\section{Tip 10: Manual therapy}

Every time we touch our patients, we must remember that the anticipation of pain can contribute to overactivtiy of the PF and subsequently contribute to more pain. Simply providing clear explanations of what interventions are recommended and why, and obtaining informed consent for assessment and treatment are important and necessary first steps. During our interventions, it is critical to move slowly, use sufficient gel, and watch and be receptive to our patients' responses. Because many of these patients have a sensitized nervous system, we need to find gentle, non-painful techniques to improve their tissue without further sensitizing them.

Physiotherapists can use various manual therapy skills to address global contribution from the back, hips, and pelvic girdle to change dysfunctional muscle patterns and/or correct asymmetry. Connective tissue treatments, such as skin rolling, will further improve their symptoms through the viserosomatic connection. Other potentially effective treatments include manual lymph drainage and visceral manipulation.

Locally, it is important to improve our patients' proprioception of their PFM's and focus on eccentric lengthening. Although OAPF are also weak, it is important not to start strengthening muscles that are overactive. Lengthening always takes precedence over strengthening. Manual techniques, such as myofacial release and scar mobilization, can help improve these structural changes and muscle imbal- 
ances. General health and mobility of the nerve pathway from S2-4 through the pelvic girdle, Alcock's canal, and down to the three separate branches of the pudendal nerve needs to be assessed and treated.

\section{Tip 11: Exercise}

Exercises that are patient-centred, fun, non-irritating, and novel will help remap the brain and have many conditioning and cardiovascular benefits. We can educate patients on how to move and pace their activities without increasing their symptoms/pain, using a graded motor imagery approach or a graded exercise approach depending on the sensitivity of their system. Body mapping exercises that focus on slow, rhythmical, and non-threatening symptom-free movement are also successful ways to help our patients move, with the ultimate goal of increasing their functional activity.

\section{Tip 12: Find what works for your patient}

Cognitive behavioural therapy (CBT) has been shown to be very effective in changing thoughts and behaviours, including catastrophization, anxiety, stress, and depression. Mindfulness has a recognized role in the treatment of chronic pain, anxiety, and the treatment of sexual dysfunction. Using any treatments that provide comfort, such as heat, ice and TENS, are useful. We must find ways to evoke a daily relaxation response to which our patients can connect. This can include mediation, yoga, Qi Gong, or Tai Chi. Encouraging social connections will help patients reintegrate back to life and can be a powerful analgesic. Encourage activities that help with positive affect, which may include gratitude training, cardiovascular exercise, meditation, and paying it forward.

Beliefs impact pain, fear impacts movement/function, and behaviour affects living. To improve our patients' quality of life, we need to address each element.

\section{Summary}

We should ensure that we are treating the entire person with a biopsychosocial, multidisciplinary approach that includes physiotherapy. By doing a thorough subjective and objective assessment, that includes relevant outcome measures, we will be able to target the right "driver" of our patients' symptoms. In all persistent pain states, there is always a blend of tissue issues and a sensitized nervous system. We need to start with pain education as the first-line treatment and make sure we are targeting all the right drivers. A multifaceted treatment approach that addresses TNE, diet, deep breathing, improved sleep hygiene, behaviour modification, and uses various manual therapy techniques and exercises, both globally and locally, can help improve the musculoskeletal contributors of our patient's symptoms. We must continue to give our patients hope and teach them self-efficacy, and put the power of their nervous system back in their hands.

Competing interests: Nelly Faghani is the co-founder of Pelvic Health Solutions.

This paper has been peer reviewed.

\section{Recommended reading}

1. FitzGerald MP, Payne CK, Lukacz ES, et al. Randomized, multicenter clinical trial of myofascial physical therapy in women with interstitial cystitis/painful bladder syndrome (IC/PBS) and pelvic floor tenderness. J Urol 2012;187:2113-8. https://doi.org/10.1016/i.juro.2012.01.123

2. The Overactive Pelvic Floor by Anna Padoa

3. Why Pelvic Pain Hurts by Adrian Louw

4. www.pelvichealthsolutions.ca for educational resources for patients and a listing on how to find a pelvic floor physiotherapists in Canada

Correspondence: Nelly Faghani, Pelvic Health Solutions, Toronto, 0N; nelly@pelvichealthsolutions.ca 